\title{
The Effect of Emotional Intelligence Training on the Quality of Life in Asthmatic Patients
}

\author{
Marzieh Sanchooli', Ghorban Fathi Aghdam², Rahim Rezai Shirazi ${ }^{3}$, Javad Sanchooli ${ }^{* *}$ \\ ${ }^{1}$ MSc Student in Psychology, Islamic Azad University, Sirjan, Iran \\ ${ }^{2}$ Faculty Member of Islamic Azad University of Abhar, Abhar, Iran \\ ${ }^{3}$ Faculty of Medicine, Golestan University of Medical Sciences, Golestan, Iran \\ ${ }^{4}$ Faculty of Medicine, Zabol University of Medical Sciences, Zabol, Iran
}

\section{*Correspondence to} Javad Sanchooli, Faculty of Medicine, Zabol University of Medical Sciences, Zabol, Iran.

Email: sanchoolij921@mums.ac.ir

Received August 4, 2016 Revised September 18, 2016 Accepted November 2, 2016 Published online November 21, 2016

\begin{abstract}
Introduction: Given the extent of asthma and its effect on physical and emotional aspects of patients' lives, the concept of emotional intelligence (EI) may propound better understanding of personal resources facilitating management of such challenges. The main objective of this study was to evaluate the effect of El training on the quality of life in patients with asthma.

Methods: This was a quasi-experimental study and 30 asthmatic patients (confirmed by clinical tests) were selected for sampling and divided into two experimental and control groups (each group containing 15 patients) randomly. The experimental group received El training in 10 sessions of 90 minutes, but the control group did not receive this training. Data gathering was done through the World Health Organization Quality of Life (WHOQOL) scales in two stages; pre- and post-test in both groups. After collecting data, they were analyzed using SPSS version 22.0.

Results: The results of the study showed that there was a significant positive correlation between $\mathrm{El}$ and quality of life, and also there was a significant difference between the mean post-test scores of experimental and control groups on measures of physical health and quality of life in asthmatic patients $(P<0.05)$.

Conclusion: According to the results achieved in this study, El can improve the quality of life in patients who suffer from asthma.

Keywords: Emotional intelligence (EI), Quality of life, Asthma, Training, Health.
\end{abstract}

\section{Introduction}

Asthma is the fourth common cause of mortality in the world in general and in industrialized countries in particular. Currently there are over 300 million asthmatic patients in the world and it is expected that this number be increased around 100 million people by the year 2025. There are approximately $13 \%$ outbreak of this disease in Iran. ${ }^{1}$ Asthma is one of the common physiological diseases that is related to a higher risk of psychological problems and detected by extensive stenosis of respiratory tracts. When the respiratory tract is prone to asthma, some stimulating psychological tensions can interact with it and cause exacerbation of dyspnea in this disease..$^{2,3}$ A study was done in 2007 to assess the relationship between the severity of symptoms and quality of life of asthmatic patients and their mental factors. Their re- sults revealed a close relationship. ${ }^{4}$ The quality of life can help the individuals to benefit a healthier and happier life. ${ }^{5}$ The quality of life in patients is a proper understanding of their position in life coming from culture and values of society and only is visible by themselves. ${ }^{6}$

Emotion or feeling is a state of mind that arises automatically rathe $r$ than through conscious effort and is often accompanied by physiological changes. Joy, grief, hate, and love are examples of emotions. ${ }^{7}$

Emotions are able to facilitate individual compatibility for adjustment in the physical and social environment. ${ }^{1}$

Mayer and Salovey in 1990 asserted that emotional intelligence (E I) is a type of emotional information pro $\mathrm{c}$ essing, including a proper assessment of emotions in their characters and others, appropriate expression of emotions, and adaptive regu-

Copyright $(2016$ The Author(s); Published by Zabol University of Medical Sciences. This is an open-access article distributed under the terms of the Creative Commons Attribution License (http://creativecommons.org/licenses/by/4.0), which permits unrestricted use, distribution, and reproduction in any medium, provided the original work is properly cited. 
lation of the emotions that enhance quality of life. ${ }^{5}$

In fact, according to this definition, the EI deals with the capacity of understanding emotions, the feelings associated with excitement, understanding their emotional information, and their management. ${ }^{8}$

Following the popularity of the concept of EI, its definition has changed fundamentally. In 1995, as defined by Goleman, EI was eligible for five domains: Knowledge of our emotions, management of our emotions, self-motivation, identifying the emotions of others, and managing relationship. According to the notion of Goleman, people in each of these domains are of different capabilities, each of them, show largely a set of habits and reply to learning. As a result, they can improve themselves by proper methods. ${ }^{9}$ A study carried out on 302 students showed that those who have a good function in the management and control of others' emotions, are supported by society and feel more satisfaction from the life..$^{10-13}$

Other research suggests that the ability to configure emotions is a key factor that might reduce stress symptoms. ${ }^{14}$ In order to confirm the positive effect of the EI set on the life satisfaction of individuals, Palmer et al used the Trait Meta-Mood Scale (TMMS). The study proved a positive correlation between two variables. ${ }^{15}$ Moreover, Saklofske et al in a study on 354 students showed that the EI has a significant positive correlation with extraversion, optimism, social acceptability, loyalty, and satisfaction of life. ${ }^{16}$ People who have a high power of adjustment in their EI, would see their events more positively and show more adaptive behavior. ${ }^{17,18}$ Most people with the power of controlling their EI have more successful countermeasures ways, because they have accurate understanding and express their emotional states properly and know how and when to express their feelings and to adjust their mood efficiently. ${ }^{19,20}$ Asthma is a common psycho-physiologic disorder characterized with extensive stenosis of airways. Psychological stressors can interact with illness and cause the aggravation of breath shortness. ${ }^{21,22}$

According to the quality of life definition provided by the World Health Organization (WHO), people's perception of their position in regard to culture and social life is based on goals, expectations, standards and priorities..$^{23,24}$ According to the researches conducted on the effect of EI over the control, regulation, and management of excitement, ${ }^{4,12,17}$ the effect of EI training was assessed on the quality of life of patients suffering from asthma in this study.

\section{Methods}

This semi-experimental study was performed in 2016. The study population included patients from age 20 to age 40 who visited the Allergy section of Prophet hospital at Golestan University of Medical Sciences. Thirty patients were identified with a clinical history of asthma based on the diagnostic allergic tests such as spirometry and lung $\mathrm{x}$-rays, and by consulting a specialist physician. Patients were as signed into test and control groups by random odd and even numbers. ${ }^{25}$
After dividing the patients into two groups of test $(n=15)$ and control $(n=15)$, both groups filled out quality of life questionnaire before beginning EI training. All patients were then participated in the pre-tests and post-tests, and only experimental group was subjected to training.

To collect data, observation, interview, and quality of life questionnaire of the WHOQOL-BREF (1996) were used in this study. Before using, the reliability and validity of the questionnaire was evaluated on Iranian general population and patients with multiple sclerosis. ${ }^{6}$

The quality of life questionnaire contained questions which measured physical health, psychological and social relationships, and environmental and living conditions. The answers were on the basis of Likert scale with five options, in the range of 1 to $5 .{ }^{26}$ Since the study was semi-experimental, before and after completion of training sessions, pre- and post-tests were carried out for both groups. This training program was established with emphasis on self-consciousness, increasing awareness, understanding, acceptance and proper expression of feelings and emotions and learning how to control them. Goleman's program on EI training was done in 8 steps including the first step: emotional perception and expression, emotional facilitation of thought, emotional understanding, emotional management; the second step: self-presentation; the third step: self-confidence; the fourth step: self-actualization; the fifth step: optimism; the sixth step: self- motivation; the seventh step: intimate relationships; and the eighth step: empathy. ${ }^{9}$ Experimental group met once per week for 2 hours up to 6 months.

After collecting, data were analyzed using SPSS version 22.0. In part of descriptive statistics, indicators of mean and standard deviation were used. In inferential statistics part, to assess the relationship between the variables, analysis of covariance and $t$ test were used. The $P$ value less than 0.05 was considered significant.

\section{Results}

To analyze data, we used descriptive and inferential statistics. Descriptive statistics included measurement table of age and education, and pre-test and post-test average, a variable measuring the quality of life in patients suffering from asthma in experimental and control groups. As inferential statistics, analysis of covariance was used in order to determine the effect of EI training on the asthmatic patients' quality of life.

Descriptive statistics showed that the data could be analyzed with parametric tests (Table 1).

Table 2 showed subscales physical health and quality of life were significant in experimental group $(P<0.05)$.

The results of analysis of covariance on the mean scores

Table 1. Demographic Characteristics of Patients

\begin{tabular}{lll}
\hline & Experiment & Control \\
\hline Age $(y)($ mean \pm SD) & $33.46 \pm 6.13$ & $32.93 \pm 7.176$ \\
Gender $(M / F)$ & $4 / 11$ & $4 / 11$ \\
Level of education $($ mean $\pm S$ D) & $2.26 \pm 2.53$ & $1.03 \pm 1.30$ \\
\hline
\end{tabular}


of post-test conducted for both experimental and control groups in the components of quality of life are summarized in Table 3.

The results suggest that EI showed a positive association with physical health and quality of life after controlling the demographic characteristics such as age, gender, and education $(P<0.05)$.

According to the results of F-test in Table 4, significant increase of components of physical health and quality of life was observed after EI training in experimental group compared to the control group $(P<0.05)$.

In the present study, we showed that EI training has the greatest impact on quality of life $\left(\mathrm{R}^{2}=0.55\right)$ and physical health $\left(R^{2}=0.41\right)$, and the lowest impact on the mental health, social relationships $\left(\mathrm{R}^{2}=0.02\right)$, and environmental health $\left(R^{2}=0.000\right)$.

Table 2. Total Score of Quality of Life and its Subscales in Experimental and Control Groups

\begin{tabular}{|c|c|c|c|c|c|}
\hline & & & Mean & SD & $P$ Value \\
\hline \multirow{4}{*}{ Physical health } & \multirow{2}{*}{ Before } & Experiment & 43.30 & 13.47 & \multirow{2}{*}{0.006} \\
\hline & & Control & 59.03 & 15.57 & \\
\hline & \multirow{2}{*}{ After } & Experiment & 65.90 & 11.13 & \multirow{2}{*}{0.344} \\
\hline & & Control & 61.04 & 16.05 & \\
\hline \multirow{4}{*}{ Quality of life } & \multirow{2}{*}{ Before } & Experiment & 49.13 & 13.71 & \multirow{2}{*}{0.029} \\
\hline & & Control & 62.43 & 17.72 & \\
\hline & \multirow{2}{*}{ After } & Experiment & 69.96 & 12.26 & \multirow{2}{*}{0.345} \\
\hline & & Control & 65.00 & 15.81 & \\
\hline \multirow{4}{*}{ Mental health } & \multirow{2}{*}{ Before } & Experiment & 50.48 & 15.34 & \multirow{2}{*}{0.215} \\
\hline & & Control & 56.53 & 10.26 & \\
\hline & \multirow[b]{2}{*}{ After } & Experiment & 70.42 & 12.51 & \multirow[b]{2}{*}{0.004} \\
\hline & & Control & 57.55 & 9.45 & \\
\hline \multirow{4}{*}{ Community relations } & \multirow{2}{*}{ Before } & Experiment & 57.65 & 15.53 & \multirow{2}{*}{0.123} \\
\hline & & Control & 66.46 & 14.82 & \\
\hline & \multirow{2}{*}{ After } & Experiment & 75.63 & 12.98 & \multirow{2}{*}{0.121} \\
\hline & & Control & 67.56 & 13.72 & \\
\hline \multirow{5}{*}{ Environmental health } & \multirow{2}{*}{ Before } & Experiment & 52.22 & 12.69 & \multirow{2}{*}{0.130} \\
\hline & & Control & 59.70 & 13.53 & \\
\hline & \multirow{3}{*}{ After } & Experiment & 66.57 & 10.04 & \multirow{3}{*}{0.200} \\
\hline & & Control & 61.04 & 12.87 & \\
\hline & & Control & 32.93 & 7.17 & \\
\hline
\end{tabular}

Table 3. Inferential Statistics and Hypotheses Testing Results $(\mathrm{n}=30)$

\begin{tabular}{|c|c|c|c|c|c|c|c|}
\hline \multirow{2}{*}{ Components } & \multicolumn{2}{|c|}{ Homogeneity of Variance } & \multicolumn{2}{|c|}{ Homogeneity of Slopes } & \multirow{2}{*}{ F Covariance } & \multirow{2}{*}{$P$} & \multirow{2}{*}{ Square Eta } \\
\hline & Levene test & $P$ & $\mathbf{F}$ & $P$ & & & \\
\hline Physical health & 3.056 & N.S & 0.962 & N.S & 19.069 & 0.000 & 0.41 \\
\hline Mental health & 2.054 & N.S & 3.179 & 0.04 & 0.643 & 0.430 & 0.02 \\
\hline Social relations & 0.05 & N.S & 0.281 & N.S & 0.010 & 0.921 & 0.00 \\
\hline Environmental health & 1.997 & N.S & 1.312 & N.S & 0.052 & 0.822 & 0.02 \\
\hline Quality of life & 0.730 & N.S & 0.961 & N.S & 33.799 & 0.000 & 0.55 \\
\hline
\end{tabular}

Table 4. Analysis of Variance for Quality of Life Scales Comparing Experimental $(\mathrm{n}=15)$ and Control Groups ( $\mathrm{n}=15)$ at Pre- and Post-Test

\begin{tabular}{|c|c|c|c|c|c|c|}
\hline Components Quality of Life & Group & Mean Difference & Standard Deviation & F-Test & $P$ & Eta-Squared \\
\hline \multirow{2}{*}{ Physical health } & Experiment & -22.60 & 12.86 & \multirow{2}{*}{39.599} & \multirow{2}{*}{0.000} & \multirow{2}{*}{0.41} \\
\hline & Control & -2.01 & 4.44 & & & \\
\hline \multirow{2}{*}{ Mental health } & Experiment & -19.94 & 8.97 & \multirow{2}{*}{716.370} & \multirow{2}{*}{0.430} & \multirow{2}{*}{0.02} \\
\hline & Control & -1.02 & 2.28 & & & \\
\hline \multirow{2}{*}{ Social relationships } & Experiment & -17.71 & 7.72 & \multirow{2}{*}{686.902} & \multirow{2}{*}{0.921} & \multirow{2}{*}{0.00} \\
\hline & Control & -1.10 & 4.40 & & & \\
\hline \multirow{2}{*}{ Environmental health } & Experiment & -14.35 & 9.86 & \multirow{2}{*}{780.079} & \multirow{2}{*}{0.921} & \multirow{2}{*}{0.00} \\
\hline & Control & -1.34 & 4.59 & & & \\
\hline \multirow{2}{*}{ Quality of life } & Experiment & -20.83 & 9.04 & \multirow{2}{*}{98.269} & \multirow{2}{*}{0.00} & \multirow[t]{2}{*}{0.55} \\
\hline & Control & -2.56 & 5.14 & & & \\
\hline
\end{tabular}




\section{Discussion}

The aim of this study was training of EI, increasing awareness, understanding, acceptance and proper expression of feelings and emotions. Moreover, asthma is one of the common diseases influenced by the psycho-physiological infectious agents, allergens and emotional states, and particularly developed serious problems for patients. As the autonomic nervous system has a close relationship with the respiratory system of asthmatics patients, and due to negative emotions, they are extremely at risk..$^{4,22}$

Training the EI can be an effective treatment for asthmatic patients based on improvement of tensions, increasing the amount of hope, and better social relationships. ${ }^{9}$ The new concept of EI may provide an experimental and theoretical framework for better understanding of individual capabilities and more effective ways of coping with health challenges and adversities. Little research is conducted on the effectiveness of EI in improving the management of disease by patients. ${ }^{27}$

The aim of present study was evaluating the positive effect of EI training on the quality of life in adult asthmatic patients. Of the most important therapeutic effects of EI training were increase of awareness, understanding, accepting, and the expression of feelings and emotions properly. People who have skills of emotion, due to their solid understanding of feelings, deal with other people and their surroundings effectively. ${ }^{24}$

Individuals, who have learned skills of controlling excitation, have their emotions guided as well. They can understand feelings of others and deal with them effectively. They are better in every area of life. ${ }^{28}$

Comparing the findings of this study based on pre-and post-test in experimental and control groups showed that EI training enhanced physical health in asthmatic patients so that significant increase could be seen completely in comparison of two groups. Moreover, researches of Ropoteanu ${ }^{29}$ and Khosrojerdi and Khan Zadeh ${ }^{30}$ showed a significant positive correlation between EI and public health.

In addition, the hypotheses of this study, that was, the effect of EI training on the quality of life in the experimental group was confirmed. Results of this study coincide with the results of Archea et al in 2007. They concede that there is a close interrelation among the quality of life, mental factors, and severity of asthma. ${ }^{4}$

Furthermore, Basinska and Drozdowska in another study on psoriatic patients showed that EI training affects the quality of life in patients. ${ }^{2}$

As well, research findings by van Leeuwen et al about the EI level in patients with vestibular schwannomas confirmed the present study. They showed that EI training has a considerable positive effect on the quality of life in these patients. ${ }^{31}$

Other findings of the present study revealed that there was not any significant difference between two groups in some of the components of quality of life (mental health, social relationships, and environmental health).

In contrast to the findings by Rahmani, ${ }^{32}$ Sharifidaramadi et $\mathrm{al},{ }^{33}$ Ismail et $\mathrm{al},{ }^{34}$ and Ehyakonandeh et al,,${ }^{35}$ this study did not approve the effect of EI training on the mental health. Currently, what is done in EI training program is raising patients' awareness about life realities, admission daring and more adaptable responses.

\section{Conclusion}

This method provides an opportunity to make individuals aware of their capabilities facing life incidences. Public culture confirms that treatment of asthma with drug is one of the limitations of research. In accordance to the variously observed attitudes among male and female patients suffering from asthma, it is suggested that the EI differences be investigated about them and its training be performed based on individuals. Moreover, because of asthma expansion, it is suggested that a similar research be done on infants suffering from this disease and this research be performed in a family area as far as possible.

\section{Ethical Approval}

This study approved by the ethical committee of Zabol University of Medical Sciences (ethical code: 63720701931036).

\section{Competing Interests}

Authors declare that they have no competing interests

\section{Acknowledgments}

This study was supported by Islamic Azad University, Sirjan, Iran. The authors of this study are grateful to Dr. Abdolghani Abdollahimohammad (faculty member of Zabol University of Medical Sciences) and to all those who cooperated in this project.

\section{References}

1. Varmaghani M, Farzadfar F, Sharifi F, et al. Prevalence of Asthma, COPD, and Chronic Bronchitis in Iran: A Systematic Review and Meta-analysis. Iran J Allergy Asthma Immunol. 2016;15(2):93-104.

2. Basinska MA, Drozdowska M. Emotional intelligence as an indicator of satisfaction with life of patients with psoriasis. Postepy Dermatol Alergol. 2013;30(6):365-372. doi:10.5114/pdia.2013.39435.

3. Goldbeck L, Koffmane K, Lecheler J, Thiessen K, Fegert JM. Disease severity, mental health, and quality of life of children and adolescents with asthma. Pediatr Pulmonol. 2007;42(1):15-22. doi:10.1002/ppul.20509.

4. Archea C, Yen IH, Chen $\mathrm{H}$, et al. Negative life events and quality of life in adults with asthma.Thorax. 2007;62:139146.

5. Mayer JD, Salovey P. Emotional Intelligence. Baywood Publishing Co; 1990:185-212.

6. Hoseinian S, Ghasemzadeh S, Niknam M. Predict the quality of life of female teachers based on variables emotional intelligence and spiritual intelligence. Quarterly Occupational and Organizational Counseling. 2011;3(9): $42-60$

7. Emotion. The American Heritage Dictionary website. 4 th ed. https://ahdictionary.com/word/search. html?q=emotion 
8. Mayer JD1, Salovey P, Caruso DR, Sitarenios G. Emotional Intelligence as a standard intelligence. Emotion. 2001;1(3):232-342.

9. Goleman D. Emotional Intelligence What It Can Matter More Than IQ. New Yourk; Bantam Book; 1995.

10. Farahbakhsh $\mathrm{S}$. The role of emotional intelligence in increasing quality of work life in school principals. Procedia - Social and Behavioral Sciences; 2012;46:31-35. doi:10.1016/j.sbspro.2012.05.062.

11. Ciarrochi J, Forgas JP, Mayer JD. Emotional intelligence in everyday life. Psychology Press Inc; 2001.

12. Ciarrochi J, Deane FP, Anderson S. Emotional intelligence moderates the relationship between stress and mental health. Pers Individ Dif. 2002;32(2):197-209. doi:10.1016/ S0191-8869(01)00012-5.

13. Nazifkar V, Bagheri M. A study on the relationship between emotional intelligence and the quality of work life in personnel of shahidrajaei port complex. Journal of Science Road. 2015;3(2):230-242.

14. Extremera N, Rey L. The moderator role of emotion regulation ability in the link between stress and well-being. Front Psychol. 2015;6:1632. doi:10.3389/fpsyg.2015.01632.

15. Palmer B, Donaldson C, Stough C. Emotional intelligence and life satisfaction. Pers Individ Dif. 2002;33(7):10911100.

16. Saklofske DH, Austin EJ, Minski PS. Factor structure and validity of a trait emotional intelligence measure. Pers Individ Dif. 2003;34(4):707-721.

17. Bekendam C. Family and dimensions of emotional intelligence. Dissertation Abstracts International. 2001;158(4):2109-2122.

18. Hasani M, Khalatbari J. The relationship between emotional intelligence and personality characteristics with the thrill-seeking in student Tonekabon in the academic year 2011-2012. Journal of Social Issues and Humanities. 2013;1(5):325-331.

19. Caruso DR, Mayer JD, Salovey P. Relation of an ability measure of emotional intelligence to personality. Journal of Personality Assessment. 2002;79(2):306-320.

20. Mayer JD, Caruso DR, Salovey P. Emotional intelligence meets traditional standards for and intelligenc. Intelligence. 1999;27:267-298.

21. Ritz T, Kullowatz A, Goldman MD, et al. Airway response to emotional stimuli in asthma: the role of the cholinergic pathway. J Appl Physiol (1985). 2010;108(6):1542-1549. doi:10.1152/japplphysiol.00818.2009

22. von Leupoldt A, Dahme B. Emotions and airway resistance in asthma: study with whole body plethysmography. Psychophysiology. 2005;42(1):92-97. doi:10.1111/j.14698986.2005.00263.x.

23. Shamili MA. Observing the relation between emotional intelligence of female teachers with performed violence against them in Bandar Abbas province. Journal of MultiDisciplinary Research. 2015;4(4):7-19.

24. Zamani SN, Bahraimian SA, Moqtaderi S, Nazari M, Hajalizadeh K. Impresment spiritual intelligence on the quality of life and psychology well-being into multiple sclerosis patients. International Journal of Biology, Pharmacy and Allied Sciences. 2015;4(9):24-35.

25. Razi E, Moosavi GA. The effect of positions on spirometric values in obese asthmatic patients. Iran J Allergy Asthma Immunol. 2007;6(3):151-154.

26. Aghapour I, Mesri M. Investigate the effects of sociocultural factors on quality of life in a group of soldiers: Based on quranic school. Journal of Quran and Medicine. 2011;1(2):23-29.

27. Zysberg L, Yosel T, Goldman M. Emotional intelligence and glycemic management among type I diabetes patients. J Health Psychol. 2015;20(7):1-6.

28. Mehmanian Khameneh M, Borjali A, Salimzadeh MK. Relationship between emotional intelligence and marital satisfaction. J Psychol. 2006;39:3.

29. Ropoteanu AC. The level of emotional intelligence for patients with bronchial asthma and a group; Rom J Intern Med. 2011;49(1):85-91.

30. Khosrojerdi $\mathrm{R}$, Khan Zadeh $\mathrm{A}$. Investigation of the relationship between emotional intelligence and public health in students of Sabzevar Tarbiat Moalm University. Journal of Sabzevar University of Medical Sciences. 2011; 14(2):110-116. [Persian].

31. van Leeuwen BM, Borst JM, Putter H, Jansen JC, van der Mey AG, Kaptein AA. Emotional intelligence in association with quality of life in patients recently diagnosed with vestibular schwannoma. Otol Neurotol. 2014;35(9):16501657. doi: $10.1097 / \mathrm{mao} .0000000000000423$.

32. Rahmani M. The effect of components of emotional intelligence training on the promotion of mental health in female athlete students; Quarterly Journal of Shahed University. 2014;1(9):75-84.

33. Sharifidaramadi P, Molavi H, Rezvani F. The effect of emotional intelligence training on general health of mothers of children with cerebral palsy in Isfahan. Knowledge \& Research in Applied Psychology. 2006;26:63-77.

34. Ismail M, Ahadi H, Delavar A, Shafiabadi A. The effect components of emotional intelligence training on mental health. Iran J Psychiatr Clin Psychol. 2007;13(2):158-165.

35. Ehyakonandeh M, Shafiabadi A, Sudani M. Evaluation of relationship between emotional intelligence and mental health in girl students of counseling at Islamic Azad University of Behbahan. Knoledge and Research in Applied Psychology. 2009;41:1-16. 